\title{
See-Thru-Gonad zebrafish line: developmental and functional validation
}

\author{
Christopher Presslauer, Teshome Tilahun Bizuayehu, Komeil Razmi, Jorge M O Fernandes \\ and Igor Babiak
}

Faculty of Biosciences and Aquaculture, Nord University, Bodø, Norway

Correspondence should be addressed to I Babiak; Email: Igor.S.Babiak@nord.no

\begin{abstract}
Zebrafish are an important model species in developmental biology. However, their potential in reproductive biology research has yet to be realized. In this study, we established See-Thru-Gonad zebrafish, a transparent line with fluorescently labeled germ cells visible throughout the life cycle, validated its gonadal development features, and demonstrated its applicability by performing a targeted gene knockdown experiment using vivo-morpholinos (VMOs). To establish the line, we crossed the $\mathrm{zf}^{45 \mathrm{Tg}}$ and $\mathrm{mitfa}^{\mathrm{w2} / \mathrm{w}^{2}}$; $m p v 17^{b 18 / b 18}$ zebrafish lines. We documented the in vivo visibility of the germline-specific fluorescent signal throughout development, from gametes through embryonic and juvenile stages up to sexual maturity, and validated gonadal development with histology. We performed targeted gene knockdown of the microRNA (miRNA) miR-92a-3p through injection of VMOs directly to maturing ovaries. After the treatment, zebrafish were bred naturally. Embryos from miR-92a-3p knockdown ovaries had a significant reduction in relative miR-92a-3p expression and a higher percentage of developmental arrest at the 1-cell stage as compared with 5-base mismatch-treated controls. The experiment demonstrates that See-Thru-Gonad line can be successfully used for vertical transmission of the effects of targeted gene knockdown in ovaries into their offspring.

Reproduction (2016) 152 507-517
\end{abstract}

\section{Introduction}

Zebrafish (Danio rerio) have undergone a remarkable journey from their origin in the rivers of India and Pakistan to the forefront of science. There are many advantages of zebrafish research including relatively low costs, short generational time, rapid embryonic development, large transparent embryos and a general robustness allowing for manipulations (Veldman \& Lin 2008). Despite this, one area of research for which the potential of zebrafish has yet to be realized is reproductive biology.

Several lines have been established in an attempt to facilitate research on reproduction in zebrafish. The zf45Tg line was created in Lisbeth Olsen's lab using the vasa regulatory regions fused with enhanced green fluorescent protein (Tg(vasa:vasa-eGFP) construct) to fluorescently label cells of the germline (Krøvel \& Olsen 2002). vasa, a conserved gene coding for a DEAD box RNA helicase, is the best known germ cell marker, with conserved and specific expression in the germline across metazoans (Gustafson \& Wessel 2010). The zf45Tg line successfully allows the tracking of primordial germ cells (PGCs) from approximately $24 \mathrm{~h}$ post fertilization (hpf) onward (Krøvel \& Olsen 2002). However, additional studies have shown that only maternal GFP signal is detected in the germline during early development, and that zygotic transcription does not begin until after sexual differentiation when the germ cells have entered meiosis around 21 days post fertilization (dpf) (Krøvel \& Olsen 2004, Leu \& Draper 2010). The $T g$ (bactin:eGFP) line was created to observe sex differentiation in zebrafish (Hsiao \& Tsai 2003). beta actin (bactin) is ubiquitously expressed throughout zebrafish but its expression is significantly higher in ovaries, allowing their identification by the contrast in signal intensity (Hsiao \& Tsai 2003). However, the $\mathrm{Tg}$ (bactin:eGFP) line cannot be used to identify gonads before $14 \mathrm{dpf}$, or testis at any stage (Hsiao \& Tsai 2003). More recently, the construct comprising promoter elements for ziwi (piwil1), the zebrafish homolog for Drosophila piwi, and eGFP, was used to create the uc1Tg line (Leu \& Draper 2010). ziwi, like vasa, is specifically expressed in the germline of zebrafish throughout development (Draper et al. 2007, Houwing et al. 2007). In this transgenic line, ziwi:eGFP is maternally supplied in embryos, while zygotic GFP can be first detected around 7 dpf (Leu \& Draper 2010). Therefore, the line is less dependent on maternal eGFP to label germ cells early in development than the zf45Tg line. However, after hatching, the usefulness of these lines in larval and subsequent development stages is limited in vivo due to interference from pigment and the general opaqueness 
of zebrafish tissue. While eGFP signal can be detected, it only allows for a rough comparison of fluorescence intensity, and does not allow for observation of gonadal structure without excision of the gonad.

In wild-type zebrafish, the pigmentation pattern consists of dark blue stripes composed of three types of pigment cells: black melanophores, yellow xanthophores and reflective iridophores (Rawls et al. 2001, White et al. 2008, Krauss et al. 2013). The tissue opaqueness can be reduced by chemical treatment, such as treating zebrafish embryos with 1-phenyl 2-thiourea before the initiation of melanogenesis (Karlsson et al. 2001). However, there is an issue with fish welfare since fish treated with 1-phenyl 2-thiourea have a marked increase in anxiety during experimental trials (Parker et al. 2013). Alternatively, some mutants such as the mitfaw2/w2; mpv17 $7^{b 18 / b 18}$ show transparent phenotypes. This line, which phenotypically resembles casper (roy -/-; nacre -/-), is a double mutant for the nacre and transparent (tra) phenotypes (White et al. 2008). The nacre phenotype is caused by a polymorphism in microphthalmia-associated transcription factor alpha (mitfa), which results in a premature stop codon (Lister et al. 1999). Zebrafish with mutant mitfa lack melanocytes completely and compensate it with an increased number of iridophores (Lister et al. 1999, White et al. 2008). The transparent phenotype is caused by a mutation in mitochondrial inner membrane protein 17 (mpv17) (Krauss et al. 2013). The mpv 17 transcript contains a splicing error between exons 2 and 3, which causes the deletion of 19 nucleotides from the open reading frame and ultimately results in a frameshift and early stop codon (Krauss et al. 2013). Transparent (-/-) zebrafish have a reduced number of iridophores and melanocytes, uniformly black pigmented eyes and a translucency of the skin (Krauss et al. 2013).

The $T g$ (bactin:eGFP) line was recently crossed with roy $(-/-)$ and albino (alb; $-/-)$ mutants to create $\beta$-ruby, a transparent and eGFP-expressing zebrafish line for use in reproductive biology (Akhter et al. 2016). While the line provides improved transparency and visualization of the ovary it contains the same limitations as the parent $T g$ (bactin:eGFP) line; gonads cannot be identified until after $14 \mathrm{dpf}$, and differentiated testis do not express eGFP signal.

miRNAs are short nonprotein-coding RNA molecules with a primary function in posttranscriptional repression of messenger RNAs (mRNAs) through associating with the miRNA-inducing silencing complex (miRISC) and then binding to the $3^{\prime}$ untranslated region of an mRNA (Bartel 2009, Fabian \& Sonenberg 2012). Depending on the complementarity between the miRNA and mRNA, the mRNA may be sequestered or degraded (Fabian \& Sonenberg 2012, Bizuayehu \& Babiak 2014). The role of miRNA during the gonadal development in fish is still largely unknown (Bizuayehu \& Babiak 2014).
Several recent studies have reported on miRNA expression in teleost gonads (Bizuayehu et al. 2012, Jing et al. 2014, Xiao et al. 2014, Vaz et al. 2015, Juanchich et al. 2016, Tao et al. 2016). However, miRNA expression during gonadal development of zebrafish, a primary teleost model, has not been profiled yet due to the small size and the challenges associated with dissecting gonads in juvenile stages.

In vivo methods for functional analysis of miRNA in zebrafish gonad have yet to be developed. Recently, targeted gene knockdown by injection of morpholino oligonucleotides antisense for an miRNA precursor was developed for the zebrafish brain (Kizil et al. 2013). There is potential that a zebrafish line optimized for reproductive biology could allow this technique to be adapted for gonadal studies as well.

The first objective of our study was to establish a stable line for use in reproductive biology with visible and fluorescently labeled germ cells throughout the zebrafish life cycle. We crossed the transgenic zf $45 \mathrm{Tg}$ and mitfa ${ }^{w 2 / w^{2}} ; m_{p v 17^{b 18 / b 18}}$ double mutant lines to create the See-Thru-Gonad line. We validated its functionality throughout gonadal development by a combination of in vivo imaging and histology. The second objective was to demonstrate the applicability of See-Thru-Gonad for miRNA functional studies. We performed targeted gene knockdown of the miRNA miR-92a-3p in sexually maturing ovaries and quantified its effects in embryos originating from the treated ovaries. miR-92a-3p was chosen for the trial as it had been shown previously to be abundant in both zebrafish gonads and embryos (Vaz et al. 2015) and had been linked to ovarian development in mammals (Liu et al. 2014, Maalouf et al. 2015); but its function had yet to be explored in teleost gonads. We demonstrated that miR92a-3p was maternally inherited and that the effect of targeted gene knockdown is vertically transmitted to the next generation.

\section{Methods}

\section{Fish}

All husbandry and experimental procedures were performed in accordance with the Norwegian Regulation on Animal Experimentation (The Norwegian Animal Protection Act, No. 73 of 20 December 1974) and were approved by the National Animal Research Authority (Utvalg for forsøk med dyr, forsøksdyrutvalget, Norway) General License for Fish Maintenance and Breeding (Godkjenning av avdeling for forsøksdyr) no. 17.

Zebrafish were maintained in an Aquatic Habitats recirculating system (Pentair, Apopka, FL, USA) following standard zebrafish procedures (Westerfield 2000). Zebrafish diet consisted of a daily mix of newly hatched Artemia nauplii (Pentair) and SDS zebrafish-specific diet (Special Diet Services, Essex, UK) following the manufacturers' recommended feeding regime. 


\section{Creation of the See-Thru-Gonad line}

The parent lines used were the zf45Tg line (Krøvel \& Olsen 2002) and the mitfaw2/w2; mpv1 $17^{\text {b18/b18 }}$ double mutant line, both obtained from The Norwegian Zebrafish Platform (zebrafish.no), Norwegian University of Life Sciences (Oslo, Norway). Initially, ten $\mathrm{F}_{0}$ breeding pairs of each line were obtained and intercrossed to become established at Nord University. For the initial hybrid crosses, zf45Tg females were spawned naturally with males of the mitfa ${ }^{w 2 / w 2}$; $m p v 17^{b 18 / b 18}$ mutant line, as only maternal eGFP signal is visible in Tg(vasa:vasa-eGFP) embryos (Krøvel \& Olsen 2002, 2004). At $2 \mathrm{dpf}$, the $F_{1}$ embryos were screened and $100 \%$ expressed the GFP signal. However, as nacre and transparent are recessive mutations, all $\mathrm{F}_{1}$ individuals were pigmented. Upon reaching sexual maturation, the $F_{1}$ fish were intercrossed, producing $3000 \mathrm{~F}_{2}$ embryos. The embryos were screened at $2 \mathrm{dpf}$ for lack of melanophores and presence of eGFP signal (Fig. 1A). The transparent mutation is not visible in embryos; therefore, the remaining fish were raised until 6 weeks post fertilization (wpf), at which time the homozygous transparent mutants could be visually identified and collected (Fig. 1B). The fish showing all three desired phenotypes were intercrossed to create the $F_{3}$ generation.

The $F_{3}$ were screened as before for the GFP signal (Fig. 1A). Because the $\mathrm{F}_{2}$ fish were heterozygotic for Tg(vasa:vasaeGFP), an estimated $75 \%$ of them were expected to express the signal. GFP-expressing fish were further reared until sexual maturation, at which time they could be screened for homozygosity of the $\operatorname{Tg}$ (vasa:vasa-eGFP). Females were crossed with $A B$ line males, and their offspring screened for GFP as before. Fish whose offspring were all expressing GFP were considered homozygous, whereas fish whose offspring were mixed were considered heterozygous (Fig. 1C). In the case of males, the transgene phenotype was observed directly in sperm (Fig. 1C). Homozygotic $\mathrm{F}_{3}$ fish were considered as a stable See-Thru-Gonad line, and their offspring were used for subsequent experiments.

\section{DNA and RNA isolation}

For DNA isolation, three-month-old See-Thru-Gonad fish were anesthetized using $50 \mathrm{mg} / \mathrm{L}$ MS-222 (Tricaine; Sigma Aldrich) buffered with equal parts of sodium bicarbonate $\left(\mathrm{NaHCO}_{3}\right)$ before caudal fin clips were taken. DNA was immediately extracted using the QuickExtract DNA Extraction Solution (Illumina, San Diego, CA, USA) according to the manufacturer's protocol.

For RNA extraction, four fish (two males and two females) were first killed with $200 \mathrm{mg} / \mathrm{L}$ buffered MS-222 followed by decapitation. Fast and slow muscle as well as skin from the trunk were excised and RNA was immediately extracted using QIAzol Lysis Reagent (Qiagen) following the manufacturer's instructions. Embryos were collected directly to QIAzol. RNA quality was assessed using 1\% (w/v) gel electrophoresis and quantified using a NanoDrop ND-1000 (Thermo Fisher Scientific, Saven \& Werner AS, Kristiansand, Norway). Total RNA was reverse transcribed using the QuantiText Reverse Transcription Kit (Qiagen) following the manufacturer's protocol.

\section{Sequencing}

To obtain genomic fragments of mpv17, mitfa, and the vasa:vasa-eGFP transgene, gene-specific primers were designed or taken from literature (Supplementary Table 1, see section on supplementary data given at the end of this article). For $m p v 17$, primers were designed to amplify a cDNA fragment as well. Polymerase chain reaction (PCR) was performed to amplify the target genes using LongAmp Taq DNA polymerase (New England BioLabs, Ipswich, MA, USA). The PCR products were excised from a $1 \%(\mathrm{w} / \mathrm{v})$ agarose gel and eluted using the QIAquick gel extraction kit (Qiagen). Amplified fragments were ligated into the pCR4-TOPO vector (Thermo Fisher Scientific) and transformed into OneShot chemically competent E. coli cells. The plasmid DNA was extracted and purified using the QIAprep Miniprep kit (Qiagen) and sequenced in both

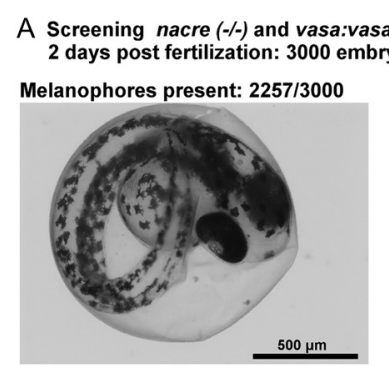

B Screening transparent $(-/-)$ 6 weeks post fertilization: 436 fish

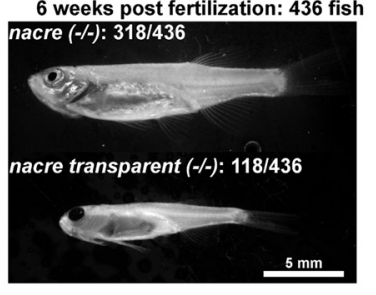

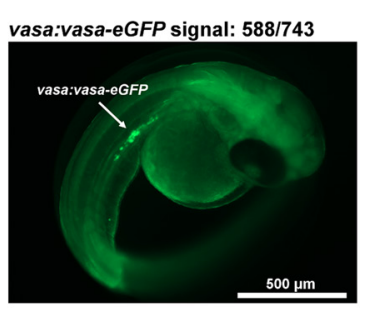

C Screening vasa:vasa-eGFP zygosity

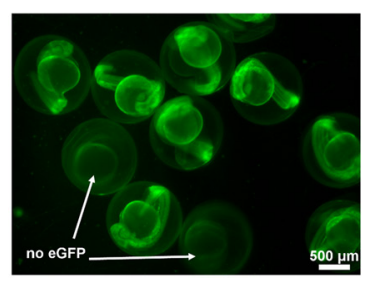

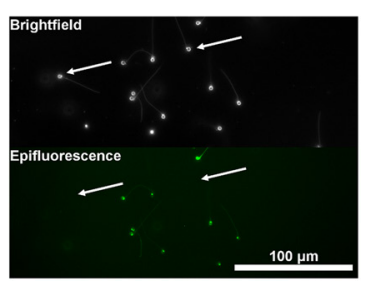

Figure 1 Screening process to create the zebrafish See-Thru-Gonad line. (A) Selection for nacre (-/-) and vasa:vasa-eGFP phenotypes at 2 days post fertilization in $\mathrm{F}_{2}$ fish. Embryos are screened for a lack of melanophores and the presence of the eGFP signal. (B) Selection for the transparent (-/-) phenotype at 6 weeks post fertilization in $F_{2}$ fish. (C) Selection of homozygosity in mature $\mathrm{F}_{3}$ fish by examination of their embryos and gametes for eGFP signal. See-Thru-Gonad females were crossed with $A B$ males and their embryos examined for eGFP signal; embryo batches with 100\% signal originated from homozygous females whereas batches with (C). Fifty percent of eGFP-positive embryos originated from heterozygotes. Spermatozoa from males were examined directly. In the picture, some spermatozoa are visible under bright field but not under epifluorescence (small arrows) - this indicates a heterozygous male. 
directions using M13 primers with the Big Dye Terminator 3.1 (Applied Biosystems) sequencing template preparation method. Sequencing reactions were analyzed by the DNA Sekvenseringslab at the University of Tromsø, Norway.

\section{Visualization of germline in vivo}

Cells of the germline were identified by their expression of the vasa:vasa-eGFP transgene (Krøvel \& Olsen 2002). Embryos at $2 \mathrm{dpf}$ were imaged directly, while larvae at $4 \mathrm{dpf}$, as well as fish at 2, 3, 6, 9 and $12 \mathrm{wpf}$ were anesthetized as described previously before in vivo imaging. Because many zebrafish diets may cause nonspecific fluorescent signal within the digestive tract, all fish at $2 \mathrm{wpf}$ and older were fasted for a minimum of $24 \mathrm{~h}$ before imaging. For gonad dissection, the zebrafish were killed as described previously and the gonad was removed under epifluorescent light using fine dissecting tools. For spermatozoa collection, mature males were anesthetized and patted dry with tissue paper before gently stroking the side of the fish. Spermatozoa were collected from the genital pore using a $10-\mu \mathrm{L}$ pipette with gentle suction and diluted in $50 \mu \mathrm{L}$ Hanks' balanced salt solution (Sigma Aldrich). All visualization of the gonads was performed using an AxioZoom V.16 microscope (Carl Zeiss) equipped with an AxioCam MRm monochrome camera (Carl Zeiss) and Zen Pro (2012; Carl Zeiss) imaging software. Images were taken using either bright field or epifluorescent light with an enhanced green fluorescent protein filter (excitation: $488 \mathrm{~nm}$, emission: $509 \mathrm{~nm}$ ).

\section{Gonadal histology}

The gonads of 3, 6, 9 and $12 \mathrm{wpf}$ zebrafish were examined. After being killed, the fish were truncated and the body cavity was opened posterior to the pectoral fins before being fixed with Bouin's solution (Sigma Aldrich) overnight at $4^{\circ} \mathrm{C}$. After fixation, the samples were embedded in paraffin, sectioned and stained using hematoxylin and eosin as described previously (Presslauer et al. 2014). At 3 wpf, before sexual differentiation, undifferentiated gonads from three individuals $(n=3)$ were examined. From $6 \mathrm{wpf}$ onward, four fish of each sex $(n=4+4)$ were examined at each time point. Staging of gonads was performed according to the previously established literature (Selman et al. 1993, Maack \& Segner 2003, Neumann et al. 2011).

\section{Knockdown of miRNA in adult zebrafish}

A VMO complementary to the dre-miR-92a-3p guide strand (5'-TACAGGCCGGGACAAGTGCAATACC-3')aswellasa5-base

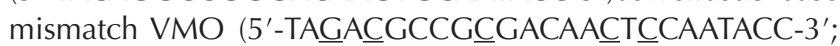
underlined letters indicate the mismatches) as a negative control were designed and produced by Gene Tools LLC (Philomath, OR, USA). The treatment was administered to 12 wpf females (Supplementary Table 2). For each injection, fish were anesthetized and injected directly to the posterior portion of the ovary using a $10.0 \mu \mathrm{L}$ syringe equipped with a $34 \mathrm{~g}$ needle (Hamilton, Bonaduz, Switzerland). Injections were either 2.0 or $3.0 \mu \mathrm{L}$ of $0.5 \mathrm{mM}$ morpholino or the mismatch control.
After 3 days of recovery, the fish were allowed to spawn naturally. From each spawning, fifteen embryos were collected and RNA was immediately extracted as described previously. The remaining embryos were monitored and developmental features were recorded at 4 and $24 \mathrm{hpf}$.

\section{Real-time quantitative RT-PCR}

Two reference genes (miR-26a-5p and miR-99a-5p) were chosen based on their lack of similarity to the targeted miR$92 a-3 p$ and their relatively high abundance in the maturing zebrafish ovary. TaqMan MicroRNA Assays (Thermo Fisher Scientific) were performed for both reference and target genes on a LightCycler 480 (Roche, Mannheim, Germany). The thermal cycle conditions used were $95^{\circ} \mathrm{C}$ for $10 \mathrm{~min}$, followed by 55 cycles of $95^{\circ} \mathrm{C}$ for $15 \mathrm{~s}, 60^{\circ} \mathrm{C}$ for $1 \mathrm{~min}$ and $72^{\circ} \mathrm{C}$ for $1 \mathrm{~s}$. The $20 \mu \mathrm{L}$ PCR consisted of $1 \times$ TaqMan small RNA assay, 30× diluted reverse transcription product and $1 \times$ TaqMan Universal PCR Master Mix II. All samples were run in duplicate with no reverse transcription and no template negative controls. Cycle threshold $\left(C_{T}\right)$ values were determined using the LightCycler 480 software cycle cut-off value of 40 . A five-point serial dilution curve (dilutions 1:15-1:240) was used to determine the efficiency of the PCR processes. Normalization between samples was performed using miR-26a-5p and miR-99a-3p as reference genes and normalization factors were calculated using geNorm (Vandesompele et al. 2002), which provided a stability value of 0.863 .

\section{Statistical analyses}

Percentage data (frequency of embryos with arrested development) were arcsine square roots transformed. The effect of treatment at two injection doses on miR-92a-3p expression and occurrence of developmental arrest in early embryos was evaluated using a two-way ANOVA. Since no significant firstorder interaction treatment $\times$ dose was found, the model was broken down and each injection dose was analyzed separately with a simple ANOVA. The effect was considered significant at $P$ value of 0.05 or less (Zar 1999).

\section{Results}

\section{Identification of See-Thru-Gonad mutations}

Sequencing of zebrafish mpv17 cDNA confirmed the deletion of 19 nucleotides within the coding sequence, resulting in a frameshift and early stop codon (Supplementary Fig. 1A) and was consistent with a previous report (Krauss et al. 2013). PCR for the corresponding genomic region using conditions optimized for an increased product size amplified a region approximately $3 \mathrm{~kb}$ in length, compared with an expected amplicon of 284 nucleotides (Supplementary Fig. 1B). Sequencing of this fragment from both ends revealed an insert of approximately $3000 \mathrm{bp}$ near the end of the second exon in the mpv17 gene (Supplementary Fig. 1C, Supplementary Table 3). Sequencing of zebrafish mitfa confirmed the polymorphism reported 

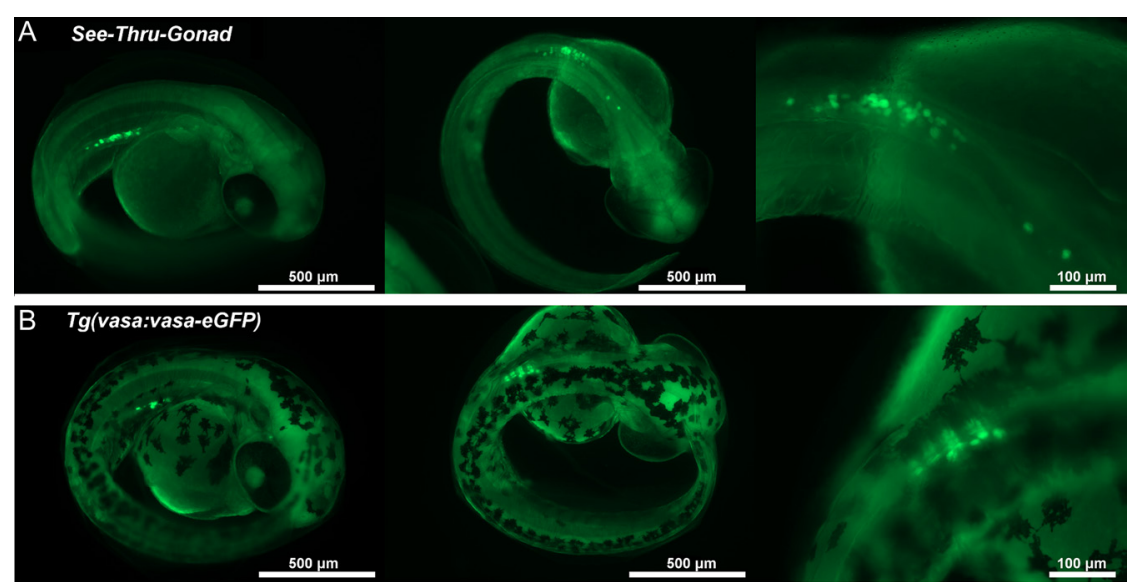

Figure 2 Comparison of primordial germ cell visibility at 2 days post fertilization between the (A) See-Thru-Gonad and (B) zf45Tg zebrafish lines. Left column - side view; center column - dorsal view; right column magnification of primordial germ cells visible at dorsal view. Primordial germ cells in See-Thru-Gonad embryos are seen in better resolution and detail. previously within exon 7 (Lister et al. 1999). This polymorphism resulted in a UAA stop codon, resulting in premature termination of translation (Supplementary Fig. 1D). In addition, the vasa:vasa-eGFP transgene was successfully amplified (Supplementary Table 3).

\section{Visualization of the germline in vivo}

At $2 \mathrm{dpf}$, the See-Thru-Gonad line showed enhanced resolution of germ cells when compared with the zf45Tg line (Fig. 2). A comparison of the lateral and dorsal views shows most PGCs to be on the same lateral plane in small clusters. When counting PGCs, an average of 26 (s.D. $=3.7, n=5$ ) could be identified in the See-Thru-Gonad line, whereas a lack of transparency made identification of individual PGCs in the zf45Tg line not possible.

At $4 \mathrm{dpf}$, germ cells were clearly visible near the gonadal anlagen (Fig. 3A). A lateral view showed the germ cells on the same lateral plane, while a dorsal view allowed observation of the gonadal structure at this time. At $2 \mathrm{wpf}$, the gonad was not identifiable using transmitted light, but imaging using epifluorescent light identified a compact gonad approximately
$0.5 \mathrm{~mm}$ in length (Fig. 3B). Germ cells were still visible individually, although some appeared to form clusters. At $3 \mathrm{wpf}$, the putative gonad was easily distinguishable using transmitted light and was further confirmed using epifluorescent light and histology. The gonad could be precisely dissected from this stage onward. The germ cells had now formed clusters composed of type I and meiotic type II germ cells and an ingrowth of stroma cells (Fig. 3C).

At $6 \mathrm{wpf}$, ovaries were easily distinguishable in live fish and were positioned between the comparatively darker digestive tract and the swim bladder (Fig. 4A). The ovary consisted predominantly of oocytes in the primary growth stage with both pre-follicle (la) and follicle phase (Ib) oocytes present. At $9 \mathrm{wpf}$, the ovaries had increased in mass and occupied the majority of the body cavity (Fig. 4B). Higher intensity eGFP signal in the ventral portion of the gonad corresponded to primary growth oocytes, whereas oocytes in the cortical alveolus stage (II) and vitellogenesis (III) were observed with increased frequency in the dorsal region. In addition, this stage was characterized by the development of the zona radiata and the formation of the theca and granulosa layers. At $12 \mathrm{wpf}$, the fish had reached sexual maturity
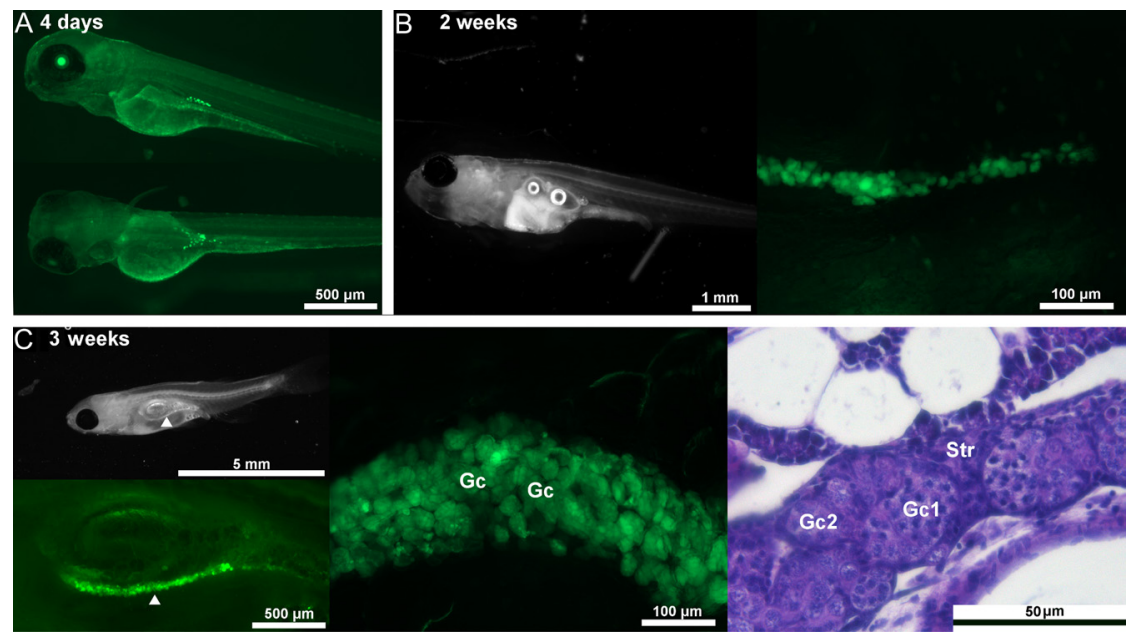

Figure 3 Visualization of the zebrafish germline before sexual differentiation. (A) 4 days post fertilization: lateral and dorsal images allow clear viewing of bright eGFP-expressing cells presumed to be germ cells. (B) 2 weeks post fertilization: the gonad is not visible under transmitted light. Increased magnification and the use of epifluorescent light reveal the proliferating germ cells. (C) 3 weeks post fertilization: the gonad is demarcated under transmitted and epifluorescent light (arrowheads).

A comparison of histology and morphology of excised gonad identifies eGFP-expressing cells to be type I (Gc1) and meiotic type II germ cells (Gc2). Str, stroma cells. 

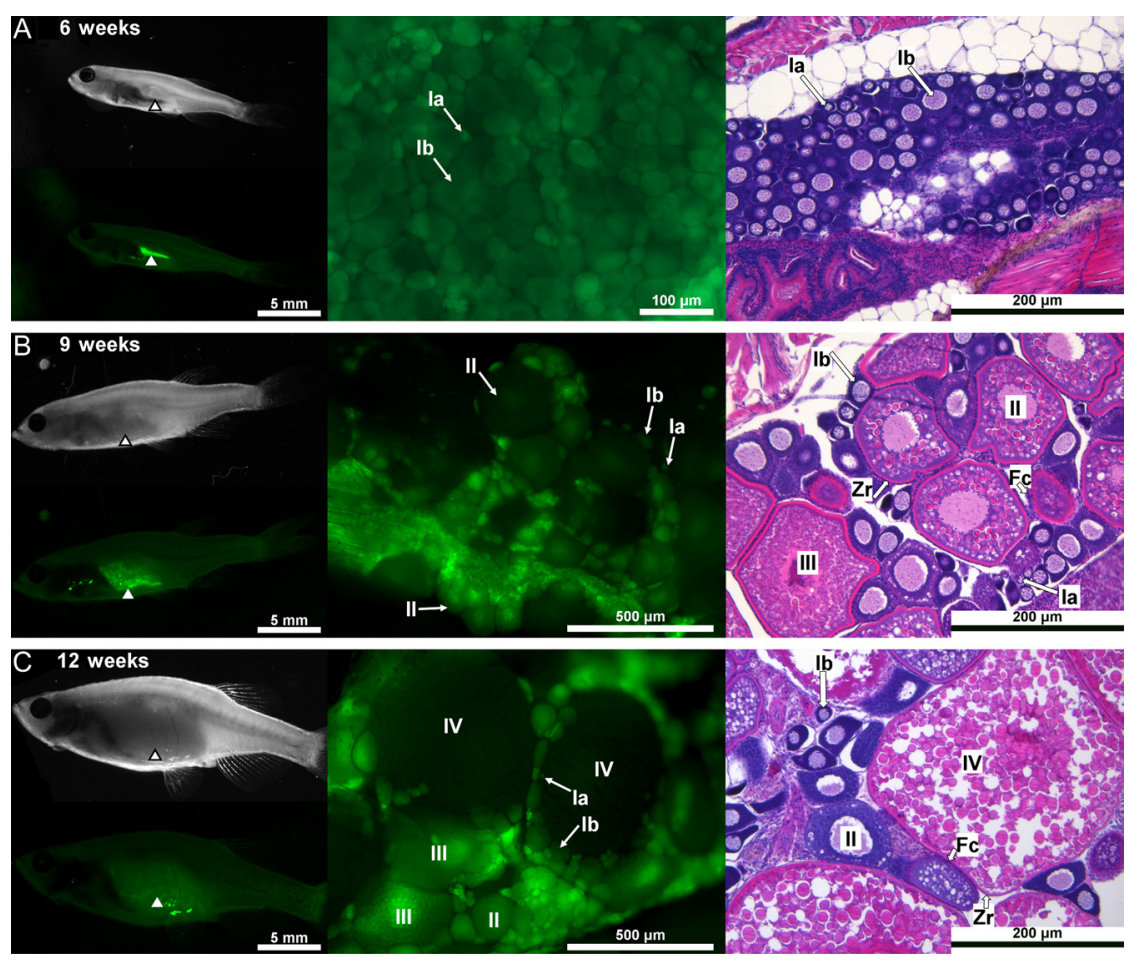

Figure 4 Visualization of the germline during ovarian development in zebrafish at 6 (A), 9 (B) and 12 (C) weeks post fertilization. Each panel consists of the whole fish displayed both using transmitted (top left) and epifluorescent (bottom left) light, the excised gonad under epifluorescent light (middle) as well as gonad histology (right). Stages of oocyte development: la - primary growth pre-follicle stage, $\mathrm{lb}$ - primary growth follicle stage, II - cortical alveolus stage,

III - vitellogenesis, IV - oocyte maturation; Fc, Follicle cells; Zr, zona radiata. Arrowheads identify the gonad in whole fish. and the body cavity was filled with vitellogenic and mature (IV) oocytes (Fig. 4C). Under epifluorescent light, vitellogenic oocytes were distinguishable by numerous lipid droplets, while mature oocytes had a comparatively dark appearance.

It was not possible to observe testis under the transmitted light during their development.
However, the GFP signal was seen in vivo under epifluorescent light (Fig. 5). At $6 \mathrm{wpf}$, the testis consisted primarily of clustered spermatogonia and spermatocytes in various stages of development (Fig. 5A). At $9 \mathrm{wpf}$, large clusters of spermatids were visible (Fig. 5B). The corresponding whole testis images showed clusters of either spermatocytes or spermatids


Figure 5 Visualization of the germline during testis development in zebrafish at 6 (A), 9 (B) and $12(\mathrm{C})$ weeks post fertilization. Each panel consists of the whole fish displayed both using transmitted (top left) and epifluorescent (bottom left), the excised gonad under epifluorescent light (middle) as well as gonad histology (right). Sg, spermatogonia; Sc, spermatocytes; St, spermatids; $\mathrm{Sp}$, spermatozoa. Arrowheads identify the gonad in whole fish. 
A
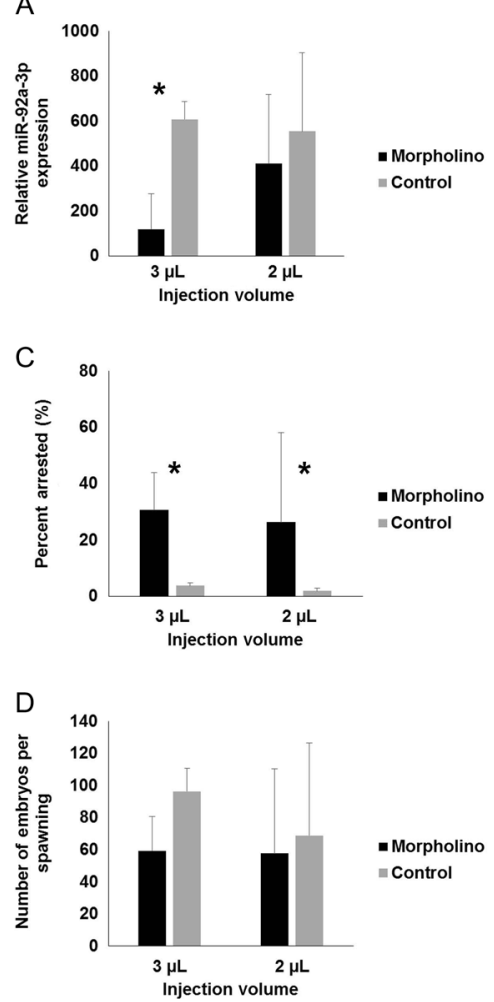

B

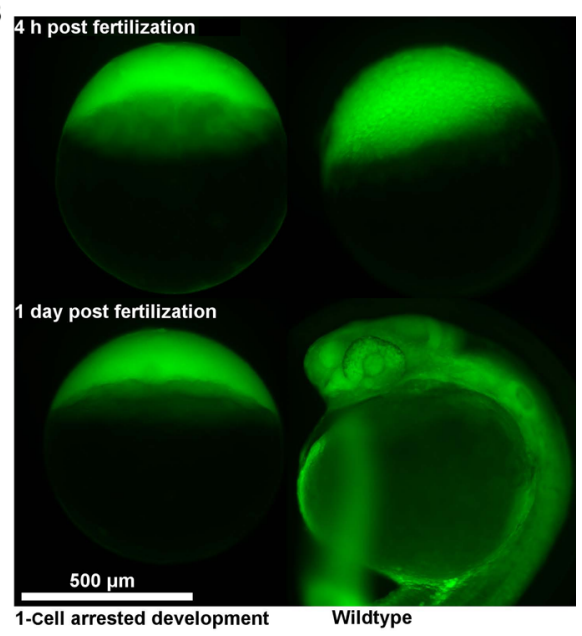

Figure 6 Effects of targeted gene knockdown using a vivo-morpholino (VMO) antisense to the microRNA miR-92a-3p injected to mature zebrafish ovaries and quantified in embryos originating from the treated females. (A) Dosedependent decrease in relative abundance of miR-92a-3p in VMO embryos, as compared with control embryos treated with a 5-base mismatch control VMO. (B) Comparison of 1-cell arrested development embryos from the miR-92a-3p VMO treatment group compared with wild-type embryos at 4 (oblong) and $24 \mathrm{~h}$ (somitogenesis) post fertilization.

(C) Comparison of the proportion of embryos arrested at 1-cell stage of development between the treatment and control groups. (D) Average number of embryos produced in different $\mathrm{VMO}$ treatment groups. Asterisks represent significant differences $(P<0.05)$. Error bars represent standard deviation.

surrounded by spermatogonia, which were defined by their comparatively bright GFP signal. At 12 wpf, males were sexually mature (Fig. 5C) and the testes were more noticeable compared with younger fish, and spermatozoa were observed among well-defined clusters of cells in various stages of spermatogenesis.

\section{Knockdown of miR-92a-3p}

The effect of the VMO treatment was dose-dependent and vertically transmitted. Injection of $0.5 \mathrm{mM} \mathrm{VMO}$ at a dose of $3.0 \mu \mathrm{L}$ directly into ovaries resulted in a significantly lower relative expression of miR-92a-3p in embryos originating from the VMO ovaries, as compared with embryos originating from ovaries receiving equivalent injections of mismatch control $\left(F_{1,4}=15.4, P=0.02\right)$. By comparison, the treatment effect was not significant $(P>0.05)$ when injecting a $2.0 \mu \mathrm{L}$ volume and the variation between batches was considerably greater (Fig. 6A).

Arrested development at the 1-cell stage was the primary phenotype associated with the treatment (Fig. 6B). On average, 30.6 $\pm 13.3 \%$ (mean \pm s.D.) and $26.4 \pm 31.8 \%$ of embryos from the 3.0 and $2.0 \mu \mathrm{L}$ dose treatment groups respectively were arrested at the 1-cell stage, whereas in control groups it was respectively $3.7 \pm 2.3 \%$ and $1.9 \pm 3.6 \%$ (Fig. 6C). In both $\mathrm{VMO}$ concentrations, the effect of treatment to ovaries, manifested in the developmental arrest frequency, was significant $\left(F_{1,4}=16.9, P=0.01\right.$ and $F_{1,10}=7.6, P=0.02$, for 3 and $2 \mu \mathrm{L}$ injection doses respectively). There were no significant effect of treatment on the number of embryos per spawning, and variation in embryos per spawning was large (Fig. 6D). Detailed summary statistics are given in Supplementary Table 2.

\section{Discussion}

In order to use zebrafish as a model for reproductive biology, we created See-Thru-Gonad, a transparent zebrafish line with a fluorescently labeled germline, visible from $1 \mathrm{dpf}$ onward. Once established and validated, the line was used to test whether the effects of targeted gene knockdown in an ovary can persist in the offspring.

\section{Genomic validation of See-Thru-Gonad mutations}

Because of the similarity between roy (-/-) and transparent (-/-) zebrafish mutants, and an uncertainty in the background of the mutations in our fish (communication with Zebrafish International Resource Center), we determined the genomic origin of mutations in See-Thru-Gonad. We confirmed that See-Thru-Gonad contains the vasa:vasa-EGFP transgene, while also being homozygous for mutations in the mitfa (nacre) and mpv 17 (transparent) genes. While the transcript from mutant mpv 17 had been sequenced previously, the researchers were unable to amplify the gene from genomic DNA (Krauss et al. 2013). Here, we demonstrate that the 
mutation is the result of an insertion of approximately $3 \mathrm{~kb}$ in size located in the second exon of mpv17 (Supplementary Fig. 1).

\section{$\operatorname{Tg}$ (vasa:vasa-EGFP) has germline-specific expression throughout development}

The See-Thru-Gonad zebrafish line expresses the $T g$ (vasa:vasa-eGFP) specifically in the germline throughout the zebrafish lifecycle, including spermatozoa (Figs 1, 2, 3, 4 and 5). During embryogenesis, and in agreement with previous observations (Krøvel \& Olsen 2002), the expression of the transgene matched the expression pattern of vasa, which is specific to the germline (Yoon et al. 1997). Post-hatch, we found the GFP signal to be restricted to the presumptive gonad, which we confirmed both by its excision and through sectioning for histology and comparing with previous studies on zebrafish gonad development (Selman et al. 1993, Maack \& Segner 2003, Neumann et al. 2011).

Two splice variants of vasa were identified previously (Krøvel \& Olsen 2004). Pre-zygotically, until approximately $3 \mathrm{wpf}$, only the shorter variant, vas- $\delta 4$, is present in the germline; after sexual differentiation, both longer, vas-l, and shorter variants are found in the ovary, while only the shorter variant is present in the testis (Krøvel \& Olsen 2004). Abundance of vas-I and vas- $\delta 4$ was related to the strength of fluorescent signal in zf45Tg fish, with the latter variant producing only a faint signal (Krøvel \& Olsen 2004). In this study, we also experienced that intensity of fluorescent signal in the male germline was weaker; nevertheless, the signal could easily be detected in the See-Thru-Gonad males.

\section{See-Thru-Gonad provides an enhanced model for in vivo viewing of the germline}

The See-Thru-Gonad line allows high-resolution imaging of the germline in vivo. During embryogenesis, PGCs actively migrate from their site of specification to the developing gonad, typically arriving within $24 \mathrm{hpf}$ to form clusters on either side of the lateral mesoderm (Yoon et al. 1997, Weidinger et al. 2002). With SeeThru-Gonad, we were able to identify individual PGCs at $2 \mathrm{dpf}$ (Fig. 2A). At this time, several of the embryos examined had individual PGCs that had yet to complete their migration but may not have been detected in zf45Tg fish due to being obscured by pigments.

Post-hatch, observations at $4 \mathrm{dpf}$ and $2 \mathrm{wpf}$ were still able to distinguish individual PGCs in vivo. During this period PGCs are proliferating, and the threshold number of PGCs formed during this period determines the future sex of zebrafish (Siegfried \& Nüsslein-Volhard 2008, Tzung et al. 2015). In the study by Tzung et al. (2015), PGC counts from excised gonads at $14 \mathrm{dpf}$ were sexually dimorphic, and showed some correlation with the respective sex ratios of their zebrafish families
(Tzung et al. 2015). Use of See-Thru-Gonad would allow for observation of PGCs in live individuals, with the ability to track the same individual through sexual maturation.

After sexual differentiation, the See-Thru-Gonad line allowed us to observe the structure of the ovary based on the relative intensity of the eGFP signal. Oocytes in the primary growth stage had noticeably higher eGFP intensity than oocytes in the cortical alveoli and vitellogenic stages, thus making it possible to evaluate the stage of ovary development in vivo (Fig. 4). In males, we were also able to in vivo detect and evaluate the testis development (Fig. 5). Although eGFP intensity was relatively low and required either a high magnification or longer exposure time than in the case of ovaries, SeeThru-Gonad would still be useful for quickly sexing fish, or locating and observing crude morphology of the testis.

These traits provide a significant improvement over the transparent $\mathrm{Tg}$ (bactin:eGFP)-based line $\beta$-ruby, which does not allow identification of germ cells in fish younger than 2 wpf or in males (Hsiao \& Tsai 2003, Akhter et al. 2016). In addition, the $T g$ (vasa:vasa-eGFP) transgene is germline-specific (Krøvel \& Olsen 2002), while GFP signal in $\beta$-ruby has been detected in the ovary, gills, as well as a small cluster of cells near the genital pore (Akhter et al. 2016).

The uc1Tg and See-Thru-Gonad lines show similar eGFP expression patterns throughout development, but they differ primarily in the initiation of zygotic transcription. While ziwi:eGFP transcription begins at $2 \mathrm{dpf}$, the vasa:vasa-eGFP signal is maternal until $3 \mathrm{wpf}$, with the initiation of meiosis (Krøvel \& Olsen 2004, Leu \& Draper 2010). In our study, we found maternal GFP sufficiently labeled proliferating germ cells before meiosis (Fig. 3). Leu and Draper (2010) demonstrated that stage la oocytes which have yet to enter the diplotene stage of meiotic prophase I do not transcribe $T g$ (vasa:vasa-eGFP) and may not be detected after the maternal signal fades. However, we suggest that See-Thru-Gonad may provide additional flexibility for researchers, since crossing See-Thru-Gonad males with mitfa ${ }^{w 2 / w 2} ; m p v 17^{b 18 / b 18}$ females would create a transparent hybrid allowing for the observation of the onset of meiosis in live fish.

\section{Morpholino injections into ovaries result in reduced miR-92a-3p expression in embryos}

In this study we demonstrated that injection of a VMO targeting miRNA miR-92a-3p directly into the zebrafish ovary resulted in significantly decreased abundance of mature miR-92a-3p in 1-cell stage embryos, as well as a significantly increased proportion of embryos with arrested development (Fig. 6). Previously, morpholinobased targeted gene knockdown of the protein-coding gene incenp was performed in oocytes of the frog (Xenopus laevis) before fertilization, but it required the excision and direct injection of the oocytes before 
transplanting them back into ovulating females (Leblond et al. 2012). To our knowledge, this study is the first demonstration of the vertical transmission of the effect of targeted knockdown of a miRNA, as well as the first time an injection of a VMO to the ovary was observed to have a vertical transmission of the effect to the subsequent embryos.

It is unclear whether the transmission of miR-92a-3p to oocytes occurred after the final maturation, or during vitellogenesis. Because morpholino oligomers are capable of targeting both mature and precursor miRNA, it would be beneficial to explore the composition of these forms of miR-92a transcripts present in oocytes during maturation. We made attempts to collect unfertilized eggs and thus measure the relative expression of mature miR-92a-3p before fertilization. Unfortunately, we were unable to collect sufficient quantities of eggs from injected fish for analysis. Regardless, our findings suggest a novel tool for examining the function of maternal miRNA early in embryonic development.

miR-92a-3p belongs to the conserved miR-17/92 cluster, which in mammals has been linked to regulation of the cell cycle, proliferation and apoptosis (Mogilyansky \& Rigoutsos 2013). In mammalian models, one function of miR-92a-3p is to regulate cell proliferation by promoting the cell cycle transition from the $\mathrm{G} 1$ to $S$ phase (Manni et al. 2009, Zhou et al. 2015). Another study has shown mammalian miR-92a-3p to regulate insulin expression and maintain glucose homeostasis (Setyowati Karolina et al. 2013). During zebrafish embryogenesis, miR-92a-3p regulates endoderm specification, left/right axis formation as well as control the BMP and nodal signaling pathways through its interaction with their antagonists (Li et al. 2011, Ning et al. 2013). However, the role of miR-92a-3p during ovary maturation and early embryo cleavage has yet to be explored.

Our findings suggest a correlation between miR92a-3p abundance and the ability of the embryo to initiate cleavage. Reasons for a zebrafish embryo failing to initiate cleavage may include a failure to complete meiosis, a lack of fertilization or a block to the initiation of the first cell cycle (Dosch et al. 2004). Some of the predicted zebrafish miR-92a-3p targets (Vejnar \& Zdobnov 2012, Agarwal et al. 2015) are highly expressed maternal genes in unfertilized oocytes and during the first cell divisions (Aanes et al. 2011), and have functions related to meiosis and the cell cycle. These include wee1 homolog 2 (wee2), cyclin a 1 (ccna1) and eukaryotic translation initiation factor $4 E$ nuclear import factor 1 (eif4enif1). In mammals, Wee2 encodes a conserved oocyte-specific meiosis inhibitor essential to maintaining oocytes in meiotic arrest (Han et al. 2005, Hanna et al. 2010, Oh et al. 2010). In the mouse (Mus musculus), Ccna 1 is expressed exclusively in the germ cell lineage and is essential for spermatocyte passage into the first meiotic division (Liu et al. 1998), while in frog, addition of cyclin A to eggs resulted in stable arrest during metaphase of the cell cycle (Roy et al. 1991). Also in mouse, Eif4enif1 is essential for the progression of meiosis, although the mechanism through which it acts is still unclear (Pfender et al. 2015). Future studies will attempt to confirm the predicted relationship between zebrafish miR-92a-3p and its predicted targets with possible functions in regulating meiosis and the cell cycle.

\section{Conclusion}

The See-Thru-Gonad line offers germline-specific signal throughout zebrafish development and enables highresolution observation of germ cell development in vivo. In addition, the application of See-Thru-Gonad as a resource for reproductive biology was demonstrated by performing targeted gene knockdown of miR-92a-3p in mature ovaries and measuring the treatment effects in embryos after natural spawning. Embryos from treated fish had significantly reduced expression of miR-92a-3p and significantly increased proportion of embryos arrested at the 1 -cell stage. For the first time, the effects of targeted gene knockdown using VMOs have been observed to have vertical transmission to offspring.

\section{Supplementary data}

This is linked to the online version of the paper at http://dx.doi. org/10.1530/REP-16-0328.

\section{Declaration of interest}

The authors declare that there is no conflict of interest that could be perceived as prejudicing the impartiality of the research reported.

\section{Funding}

This study was financed by the Research Council of Norway (FishmiR project \# 213825) and Nord University.

\section{Acknowledgements}

We are grateful to Professor Peter Aleström (Norwegian University of Life Sciences, Oslo) for providing the zebrafish lines used in the experiment and to Dr Lisbeth Olsen (University of Bergen, Norway) for kindly sharing the zf45Tg line. In addition, we thank Cesilie Amundsen and Katrine Klippenberg for their assistance in breeding and maintaining the See-Thru-Gonad line.

\section{References}

Aanes H, Winata CL, Lin CH, Chen JP, Srinivasan KG, Lee SGP, Lim AYM, Hajan HS, Collas P, Bourque G et al. 2011 Zebrafish mRNA sequencing deciphers novelties in transcriptome dynamics during maternal to zygotic transition. Genome Research 21 1328-1338. (doi:10.1101/ gr.116012.110) 
Agarwal V, Bell GW, Nam J-W \& Bartel DP 2015 Predicting effective microRNA target sites in mammalian mRNAs. elife 4 e05005. (doi:10.7554/elife.05005)

Akhter A, Kumagai R-I, Roy SR, Ii S, Tokumoto M, Hossain B, Wang J, Klangnurak W, Miyazaki T \& Tokumoto T 2016 Generation of transparent zebrafish with fluorescent ovaries: a living visible model for reproductive biology. Zebrafish 13 155-160. (doi:10.1089/zeb.2015.1116)

Bartel DP 2009 MicroRNA target recognition and regulatory functions. Cell 136 215-233. (doi:10.1016/j.cell.2009.01.002)

Bizuayehu TT \& Babiak I 2014 MicroRNA in teleost fish. Genome Biology and Evolution 6 1911-1937. (doi:10.1093/gbe/evu151)

Bizuayehu TT, Babiak J, Norberg B, Fernandes JMO, Johansen SD \& Babiak I 2012 Sex-biased miRNA expression in atlantic halibut (Hippoglossus hippoglossus) brain and gonads. Sexual Development 6 257-266. (doi:10.1159/000341378)

Dosch R, Wagner DS, Mintzer KA, Runke G, Wiemelt AP \& Mullins MC 2004 Maternal control of vertebrate development before the midblastula transition. Developmental Cell 6 771-780. (doi:10.1016/j. devcel.2004.05.002)

Draper BW, McCallum CM \& Moens CB 2007 nanos1 is required to maintain oocyte production in adult zebrafish. Developmental Biology 305 589-598. (doi:10.1016/j.ydbio.2007.03.007)

Fabian MR \& Sonenberg N 2012 The mechanics of miRNA-mediated gene silencing: a look under the hood of miRISC. Nature Structural and Molecular Biology 19 586-593. (doi:10.1038/nsmb.2296)

Gustafson EA \& Wessel GM 2010 Vasa genes: emerging roles in the germ line and in multipotent cells. BioEssays 32 626-637. (doi:10.1002/ bies.201000001)

Han SJ, Chen R, Paronetto MP \& Conti M 2005 Wee1B is an oocytespecific kinase involved in the control of meiotic arrest in the mouse. Current Biology 15 1670-1676. (doi:10.1016/j.cub.2005.07.056)

Hanna CB, Yao S, Patta MC, Jensen JT \& Wu X 2010 WEE2 is an oocytespecific meiosis inhibitor in rhesus macaque monkeys. Biology of Reproduction 82 1190-1197. (doi:10.1095/biolreprod.109.081984)

Houwing S, Kamminga LM, Berezikov E, Cronembold D, Girard A, van den Elst H, Filippov DV, Blaser H, Raz E, Moens CB et al. 2007 A role for Piwi and piRNAs in germ cell maintenance and transposon silencing in zebrafish. Cell 129 69-82. (doi:10.1016/j.cell.2007.03.026)

Hsiao C-D \& Tsai H-J 2003 Transgenic zebrafish with fluorescent germ cell: a useful tool to visualize germ cell proliferation and juvenile hermaphroditism in vivo. Developmental Biology 262 313-323. (doi:10.1016/S0012-1606(03)00402-0)

Jing J, Wu J, Liu W, Xiong S, Ma W, Zhang J, Wang W, Gui J-F \& Mei J 2014 Sex-biased miRNAs in gonad and their potential roles for testis development in yellow catfish. PLOS ONE 9 e107946. (doi:10.1371/ journal.pone.0107946)

Juanchich A, Bardou P, Rué O, Gabillard J-C, Gaspin C, Bobe J \& Guiguen Y 2016 Characterization of an extensive rainbow trout miRNA transcriptome by next generation sequencing. BMC Genomics 17 1-12. (doi:10.1186/s12864-015-2294-6)

Karlsson J, von Hofsten J \& Olsson P-E 2001 Generating transparent zebrafish: a refined method to improve detection of gene expression during embryonic development. Marine Biotechnology 3 522-527. (doi:10.1007/s1012601-0053-4)

Kizil C, Iltzsche A, Kaslin J \& Brand M 2013 Micromanipulation of gene expression in the adult zebrafish brain using cerebroventricular microinjection of morpholino oligonucleotides. Journal of Visualized Experiments e50415. (doi:10.3791/50415)

Krauss J, Astrinides P, Frohnhöfer HG, Walderich B \& Nüsslein-Volhard C 2013 Transparent, a gene affecting stripe formation in zebrafish, encodes the mitochondrial protein Mpv17 that is required for iridophore survival. Biology Open 2 703-710. (doi:10.1242/bio.20135132)

Krøvel AV \& Olsen LC 2002 Expression of a vas::EGFP transgene in primordial germ cells of the zebrafish. Mechanisms of Development 116 141-150. (doi:10.1016/s0925-4773(02)00154-5)

Krøvel AV \& Olsen LC 2004 Sexual dimorphic expression pattern of a splice variant of zebrafish vasa during gonadal development. Developmental Biology 271 190-197. (doi:10.1016/j.ydbio.2004.04.004)

Leblond GG, Sarazin H, Li R, Suzuki M, Ueno N \& Liu XJ 2012 Translation of incenp during oocyte maturation is required for embryonic development in Xenopus laevis. Biology of Reproduction 86 161. (doi:10.1095/ biolreprod.111.097972)
Leu DH \& Draper BW 2010 The ziwi promoter drives germlinespecific gene expression in zebrafish. Developmental Dynamics 239 2714-2721. (doi:10.1002/dvdy.22404)

Li N, Wei C, Olena AF \& Patton JG 2011 Regulation of endoderm formation and left-right asymmetry by miR-92 during early zebrafish development. Development 138 1817-1826. (doi:10.1242/dev.056697)

Lister JA, Robertson CP, Lepage T, Johnson SL \& Raible DW 1999 nacre encodes a zebrafish microphthalmia-related protein that regulates neural-crest-derived pigment cell fate. Development 126 3757-3767.

Liu D, Matzuk MM, Sung WK, Guo Q, Wang P \& Wolgemuth DJ 1998 Cyclin A1 is required for meiosis in the male mouse. Nature Genetics $\mathbf{2 0}$ 377-380. (doi:10.1038/3855)

Liu J, Yao W, Yao Y, Du X, Zhou J, Ma B, Liu H, Li Q \& Pan Z 2014 MiR92a inhibits porcine ovarian granulosa cell apoptosis by targeting Smad7 gene. FEBS Letters 588 4497-4503. (doi:10.1016/j.febslet.2014.10.021)

Maack G \& Segner H 2003 Morphological development of the gonads in zebrafish. Journal of Fish Biology 62 895-906. (doi:10.1046/j.10958649.2003.00074.x)

Maalouf SW, Liu WS \& Pate JL 2015 MicroRNA in ovarian function. Cell and Tissue Research 363 7-18. (doi:10.1007/s00441-015-2307-4)

Manni I, Artuso S, Careccia S, Rizzo MG, Baserga R, Piaggio G \& Sacchi A 2009 The microRNA miR-92 increases proliferation of myeloid cells and by targeting p 63 modulates the abundance of its isoforms. FASEB Journal 23 3957-3966. (doi:10.1096/fj.09-131847)

Mogilyansky E \& Rigoutsos I 2013 The miR-17/92 cluster: a comprehensive update on its genomics, genetics, functions and increasingly important and numerous roles in health and disease. Cell Death and Differentiation 20 1603-1614. (doi:10.1038/cdd.2013.125)

Neumann JC, Chandler GL, Damoulis VA, Fustino NJ, Lillard K, Looijenga L, Margraf L, Rakheja D \& Amatruda JF 2011 Mutation in the type IB bone morphogenetic protein receptor alk6b impairs germcell differentiation and causes germ-cell tumors in zebrafish. PNAS 108 13153-13158. (doi:10.1073/pnas.1102311108)

Ning G, Liu X, Dai M, Meng A \& Wang Q 2013 MicroRNA-92a upholds Bmp signaling by targeting noggin3 during pharyngeal cartilage formation. Developmental Cell 24 283-295. (doi:10.1016/j. devcel.2012.12.016)

Oh JS, Han SJ \& Conti M 2010 Wee1B, Myt1, and Cdc25 function in distinct compartments of the mouse oocyte to control meiotic resumption. Journal of Cell Biology 188 199-207. (doi:10.1083/jcb.200907161)

Parker MO, Brock AJ, Millington ME \& Brennan CH 2013 Behavioral phenotyping of casper mutant and 1-pheny-2-thiourea treated adult zebrafish. Zebrafish 10 466-471. (doi:10.1089/zeb.2013.0878)

Pfender S, Kuznetsov V, Pasternak M, Tischer T, Santhanam B \& Schuh M 2015 Live imaging RNAi screen reveals genes essential for meiosis in mammalian oocytes. Nature 524 239-242. (doi:10.1038/nature14568)

Presslauer C, Nagasawa K, Dahle D, Babiak J, Fernandes JMO \& Babiak I 2014 Induced autoimmunity against gonadal proteins affects gonadal development in juvenile zebrafish. PLOS ONE 9 e114209. (doi:10.1371/ journal.pone.0114209)

Rawls JF, Mellgren EM \& Johnson SL 2001 How the zebrafish gets its stripes. Developmental Biology 240 301-314. (doi:10.1006/dbio.2001.0418)

Roy LM, Swenson KI, Walker DH, Gabrielli BG, Li RS, Piwnica-Worms H \& Maller JL 1991 Activation of p34 ${ }^{\text {cdc2 }}$ kinase by cyclin A. Journal of Cell Biology 113 507-514. (doi:10.1083/jcb.113.3.507)

Selman K, Wallace RA, Sarka A \& Qi X 1993 Stages of oocyte development in the zebrafish, Brachydanio rerio. Journal of Morphology 218 203-224. (doi:10.1002/jmor.1052180209)

Setyowati Karolina D, Sepramaniam S, Tan HZ, Armugam A \& Jeyaseelan K 2013 miR-25 and miR-92a regulate insulin I biosynthesis in rats. RNA Biology 10 1365-1378. (doi:10.4161/rna.25557)

Siegfried KR \& Nüsslein-Volhard C 2008 Germ line control of female sex determination in zebrafish. Developmental Biology 324 277-287. (doi:10.1016/j.ydbio.2008.09.025)

Tao W, Sun L, Shi H, Cheng Y, Jiang D, Fu B, Conte MA, Gammerdinger WJ, Kocher TD \& Wang D 2016 Integrated analysis of miRNA and mRNA expression profiles in tilapia gonads at an early stage of sex differentiation. BMC Genomics 17 1-13. (doi:10.1186/s12864-015-2294-6)

Tzung K-W, Goto R, Saju Jolly M, Sreenivasan R, Saito T, Arai K, Yamaha E, Hossain Mohammad S, Calvert Meredith E \& Orbán L 2015 Early depletion of primordial germ cells in zebrafish promotes testis formation. Stem Cell Reports 4 61-73. (doi:10.1016/j.stemcr.2014.10.011) 
Vandesompele J, De Preter K, Pattyn F, Poppe B, Van Roy N, De Paepe A \& Speleman F 2002 Accurate normalization of real-time quantitative RT-PCR data by geometric averaging of multiple internal control genes. Genome Biology 3 research0034.0031-research0034.0011.

Vaz C, Wee CW, Lee GPS, Ingham PW, Tanavde V \& Mathavan S 2015 Deep sequencing of small RNA facilitates tissue and sex associated microRNA discovery in zebrafish. BMC Genomics 16 950. (doi:10.1186/ s12864-015-2135-7)

Vejnar CE \& Zdobnov EM 2012 miRmap: comprehensive prediction of microRNA target repression strength. Nucleic Acids Research 40 11673-11683. (doi:10.1093/nar/gks901)

Veldman MB \& Lin S 2008 Zebrafish as a developmental model organism for pediatric research. Pediatric Research 64 470-476. (doi:10.1203/ PDR.0b013e318186e609)

Weidinger G, Wolke U, Köprunner M, Thisse C, Thisse B \& Raz E 2002 Regulation of zebrafish primordial germ cell migration by attraction towards an intermediate target. Development 129 25-36.

Westerfield M 2000 The Zebrafish Book. A Guide for the Laboratory Use of Zebrafish (Danio rerio), 4th ed. Eugene, OR, USA: University of Oregon Press.

White RM, Sessa A, Burke C, Bowman T, LeBlanc J, Ceol C, Bourque C, Dovey M, Goessling W, Burns CE et al. 2008 Transparent adult zebrafish as a tool for in vivo transplantation analysis. Cell Stem Cell 2 183-189. (doi:10.1016/j.stem.2007.11.002)

Xiao J, Zhong H, Zhou Y, Yu F, Gao Y, Luo Y, Tang Z, Guo Z, Guo E, Gan X et al. 2014 Identification and characterization of microRNAs in ovary and testis of nile tilapia (Oreochromis niloticus) by using solexa sequencing technology. PLoS ONE 9 e86821. (doi:10.1371/journal.pone.0086821)

Yoon C, Kawakami K \& Hopkins N 1997 Zebrafish vasa homologue RNA is localized to the cleavage planes of 2- and 4-cell-stage embryos and is expressed in the primordial germ cells. Development 124 3157-3165.

Zar JH 1999 Biostatistical Analysis, 4th ed. New Jersey, USA: Prentice Hall.

Zhou C, Shen L, Mao L, Wang B, Li Y \& Yu H 2015 miR-92a is upregulated in cervical cancer and promotes cell proliferation and invasion by targeting FBXW7. Biochemical and Biophysical Research Communications 458 63-69. (doi:10.1016/j.bbrc.2015.01.066)

Received 16 June 2016

First decision 18 July 2016

Revised manuscript received 8 August 2016

Accepted 30 August 2016 